\title{
Particulate and Microbial Contamination in In-Use Admixed Intravenous Infusions
}

\author{
Katsuhiro Yorioka, ${ }^{a}$ Shigeharu Oie, ${ }^{b}$ Masafumi Oomaki, ${ }^{a}$ Akihisa Imamura, ${ }^{a}$ and Akira KamiYA ${ }^{*}, b$ \\ ${ }^{a}$ Department of Pharmacy, Saiseikai Yamaguchi General Hospital; 2-11 Midoricho, Yamaguchi 753-0078, Japan: and \\ ${ }^{b}$ Department of Pharmacy, Yamaguchi University Hospital; 1-1-1 Minamikogushi, Ube 755-8505, Japan. \\ Received May 19, 2006; accepted August 17, 2006; published online August 23, 2006
}

We compared particulate and microbial contamination in residual solutions of peripheral intravenous admixtures after the termination of drip infusion between intravenous fluids admixed with glass ampoule drugs and those admixed with pre-filled syringe drugs. The mean number of particles $\geq 1.3 \mu \mathrm{m}$ in diameter per $1 \mathrm{ml}$ of residual solution was 758.4 for fluids $(n=60)$ admixed with potassium chloride in a glass ampoule $(20 \mathrm{ml}$ volume), 158.6 for fluids $(n=63)$ admixed with potassium chloride in a pre-filled syringe $(20 \mathrm{ml}$ volume $), 736.5$ for fluids $(n=66)$ admixed with sodium chloride in a glass ampoule $(20 \mathrm{ml}$ volume $), 179.2$ for fluids $(n=15)$ admixed with sodium chloride in a pre-filled syringe $(20 \mathrm{ml}$ volume), 1884.5 in fluids $(n=30)$ admixed with dobutamine hydrochloride in 3 glass ampoules $(5 \mathrm{ml}$ volume), and $178.9(n=10)$ in diluted dobutamine hydrochloride in prefilled syringes $(50 \mathrm{ml}$ volume: For these samples alone, particulate and microbial contamination were evaluated in sealed products.) Thus, for potassium chloride or sodium chloride for injection, the number of particles $\geq 1.3 \mu \mathrm{m}$ in diameter in the residual intravenous solution was significantly higher for fluids admixed with glass ampoule drugs than for those admixed with pre-filled syringe drugs $(p<0.0001)$. For dobutamine hydrochloride for injection, the number of particles $\geq 1.3 \mu \mathrm{m}$ in diameter in the residual intravenous solution was estimated to be higher for fluids admixed with its glass ampoule drug than for those admixed with its pre-filled syringe drug. Observation of the residual solutions of fluids admixed with potassium chloride, sodium chloride, or dobutamine hydrochloride in glass ampoules using an electron microscope with an X-ray analyzer showed glass fragments in each residual solution. Therefore, for the prevention of glass particle contamination in peripheral intravenous admixtures, the use of pre-filled syringe drugs may a useful method. No microbial contamination was observed in any of the residual solutions of 5 types of admixture.

Key words intravenous infusion; admixture; glass ampoule; pre-filled syringe; contamination; glass

Glass particles from glass ampoules not only causes phlebitis but also gives damage to the lungs, brain, kidneys, liver, and spleen. ${ }^{1-7)}$ Therefore, in western countries, the use of glass ampoule drugs is minimized, and vial drugs are used. However, in Asian countries such as Japan, glass ampoule drugs are still frequently used.

In recent years, pre-filled syringe drugs, which are expected to be free of glass fragments, have become commercially available in Japan. However, there have been no clinical data on the mixture of particles in pre-filled syringe drugs. Therefore, we collected residual solutions of peripheral infusions administered to patients, and compared the number of particles in the residual solutions between fluids admixed with glass ampoule drugs and those admixed with pre-filled syringe drugs. In addition, microbial contamination in these residual solutions was also examined.

\section{MATERIALS AND METHODS}

We evaluated particulate and microbial contamination in a total of 300 samples of residual solutions of peripheral infusions administered at the intensive care unit ( 6 beds) of Saiseikai Yamaguchi General Hospital (310 bed) in Yamaguchi Prefecture, Japan. The following 6 types of peripheral infusions were examined: electrolyte solution $(500 \mathrm{ml}$ volume: $n=60$ ) admixed with potassium chloride in a glass ampoule (K.C.L. ${ }^{\circledR}, 20 \mathrm{ml}$ volume), electrolyte solution (500 $\mathrm{ml}$ volume: $n=63$ ) admixed with potassium chloride in a pre-filled syringe (Mediject ${ }^{\circledR} \mathrm{K}, 20 \mathrm{ml}$ volume), electrolyte solution (500 ml volume: $n=66$ ) admixed with sodium chloride in a glass ampoule (Conclyte ${ }^{\circledR}-\mathrm{Na}, 20 \mathrm{ml}$ volume), electrolyte solution (500 ml volume: $n=15)$ admixed with sodium chloride in a pre-filled syringe (Mediject ${ }^{\circledR} \mathrm{Na} 10 \%, 20 \mathrm{ml}$ volume), physiological saline (Physisalz ${ }^{\circledR}-\mathrm{PL}, 100 \mathrm{ml}$ volume) admixed with dobutamine hydrochloride in 3 ampoules (Dobutrex $^{\circledR}, 5 \mathrm{ml}$ volume: $n=30$ ), and diluted dobutamine hydrochloride in pre-filled syringes (Dobupum ${ }^{\circledR}$ Injection $0.3 \%$ syringe, $50 \mathrm{ml}$ volume: $n=10$ ). Since dobutamine hydrochloride in pre-filled syringes is administered using a syringe pump, the recovery of residual solutions of administration was difficult, and sealed products were examined. Electrolyte solutions used for admixture were Otsuka Glucose Injection 5\%, Potacol ${ }^{\circledR}-\mathrm{R}$, Solita ${ }^{\circledR}-\mathrm{T}$ No. 3, Physio ${ }^{\circledR} 35$, and Veen- ${ }^{\circledR}$ D. As controls, particulate and microbial contamination of these electrolyte solutions $(500 \mathrm{ml}$ volume: $n=56)$ and physiological solution (Physisalz ${ }^{\circledR}-\mathrm{PL}, 100 \mathrm{ml}$ volume: $n=10)$ were also examined.

The size and number of particles were measured using a light blockage particle counter KL-04 (Rion K.K., Tokyo, Japan). In addition, the volume of the residual solution in the bag was measured. For 2 samples each of the 5 types of intravenous admixture and diluted dobutamine hydrochloride in pre-filled syringes (total, 6 types), each residual solution $(5 \mathrm{ml})$ was passed through $0.22 \mu \mathrm{m}$ membrane filters, $5 \mathrm{~cm}$ in diameter (Nippon Becton Dickinson Co., Tokyo, Japan), and the particles on this filter were observed and identified using a scanning electron microscope JSM-5600LV coupled to an energy dispersion spectroscope JEO-2200 (JSM, Tokyo, Japan).

Microorganisms were quantified by the filter filtration 
method. The residual solution $(5 \mathrm{ml})$ in the bag was passed through $0.22 \mu \mathrm{m}$ membrane filters ( $5 \mathrm{~cm}$ in diameter), and $100 \mathrm{ml}$ sterile physiological solution was passed through the filters to eliminate carry-overs such as sugar and amino acid on the filter. The filters were placed on Trypticase soy agar II with 5\% sheep blood (Nippon Becton Dickinson Co., Tokyo, Japan) and incubated for $1-7 \mathrm{~d}$ at $30^{\circ} \mathrm{C}$.

The number of particles in the residual solution was compared according to the particle size $(\geq 1.3 \mu \mathrm{m}, \geq 5 \mu \mathrm{m}, \geq 10$ $\mu \mathrm{m}, \geq 50 \mu \mathrm{m}$ ) between fluids admixed with potassium chloride in a glass ampoule and those admixed with potassium chloride in a pre-filled syringe and between fluids admixed with sodium hydrochloride in a glass ampoule and those admixed with sodium hydrochloride in a pre-filled syringe by the Wilcoxon rank sum test, and $p$ values were obtained.

\section{RESULTS}

The mean volume of residual solution samples of intra- venous infusions was $22 \mathrm{ml}$ for electrolyte solution $(500 \mathrm{ml}$ volume) admixed with drugs and $10 \mathrm{ml}$ for physiological solution ( $100 \mathrm{ml}$ volume) admixed with drugs. Table 1 shows the diameter and number of particles in 244 samples. The number of particles $\geq 1.3 \mu \mathrm{m}$ in diameter was significantly higher in fluids admixed with potassium chloride in a glass ampoule than those admixed with this drug in a pre-filled syringe $(p<0.0001)$. The number of particles $\geq 1.3 \mu \mathrm{m}$ in diameter was significantly higher in fluids admixed with sodium chloride in a glass ampoule than those admixed with this drug in a pre-filled syringe $(p<0.0001)$. The number of particles $\geq 1.3 \mu \mathrm{m}$ in diameter was estimated to be higher in fluids admixed with dobutamine hydrochloride in glass ampoules than in those admixed with this drug in pre-filled syringes. The mean number (range) of particles $\geq 1.3 \mu \mathrm{m}$ in electrolyte solution (500 $\mathrm{ml}$ volume) and physiological solution $(100 \mathrm{ml}$ volume) as controls was $95.9(58-130) / \mathrm{ml}$ for Otsuka Glucose Injection 5\% $(n=13), 109.1(86-160) / \mathrm{ml}$ for Potacol $^{\mathbb{B}}-\mathrm{R}(n=10), 112.5(61-160) / \mathrm{ml}$ for Solita ${ }^{\circledR}-\mathrm{T}$

Table 1. Mean (Range) of Particles/ml in In-Use Various Intravenous Infusion

\begin{tabular}{|c|c|c|c|c|}
\hline \multirow{2}{*}{ Infusion } & \multicolumn{4}{|c|}{ Particles greater than } \\
\hline & $1.3 \mu \mathrm{m}$ & $5 \mu \mathrm{m}$ & $10 \mu \mathrm{m}$ & $50 \mu \mathrm{m}$ \\
\hline Fluids admixed with potassium chloride in a glass ampoule $(n=60)$ & $758.4 *(388-1560)$ & $20.9 *(5-44)$ & $9.9 *(2-43)$ & $2.9 *(0-9)$ \\
\hline Fluids admixed with potassium chloride in a pre-filled syringe $(n=63)$ & $158.6(98-299)$ & $6.5(1-22)$ & $1.8(0-11)$ & 0 \\
\hline Fluids admixed with sodium chloride in a glass ampoule $(n=66)$ & $736.1 *(379-1009)$ & $23.9 *(9-65)$ & $10.6 *(2-28)$ & $2.5^{*}(0-6)$ \\
\hline Fluids admixed with sodium chloride in a pre-filled syringe $(n=15)$ & $179.2(128-245)$ & $7.6(3-16)$ & $3.2(0-7)$ & 0 \\
\hline Fluids admixed with dobutamine hydrochloride in glass ampoules $(n=30)$ & $1884.5(1001-3890)$ & $29.4(15-56)$ & $11.1(2-30)$ & $3.2(1-8)$ \\
\hline Dobutamine hydrochloride in pre-filled syringes $(n=10)$ & $178.9(160-199)$ & $2.6(0-6)$ & 0 & 0 \\
\hline
\end{tabular}

$* p<0.0001$.

(a)
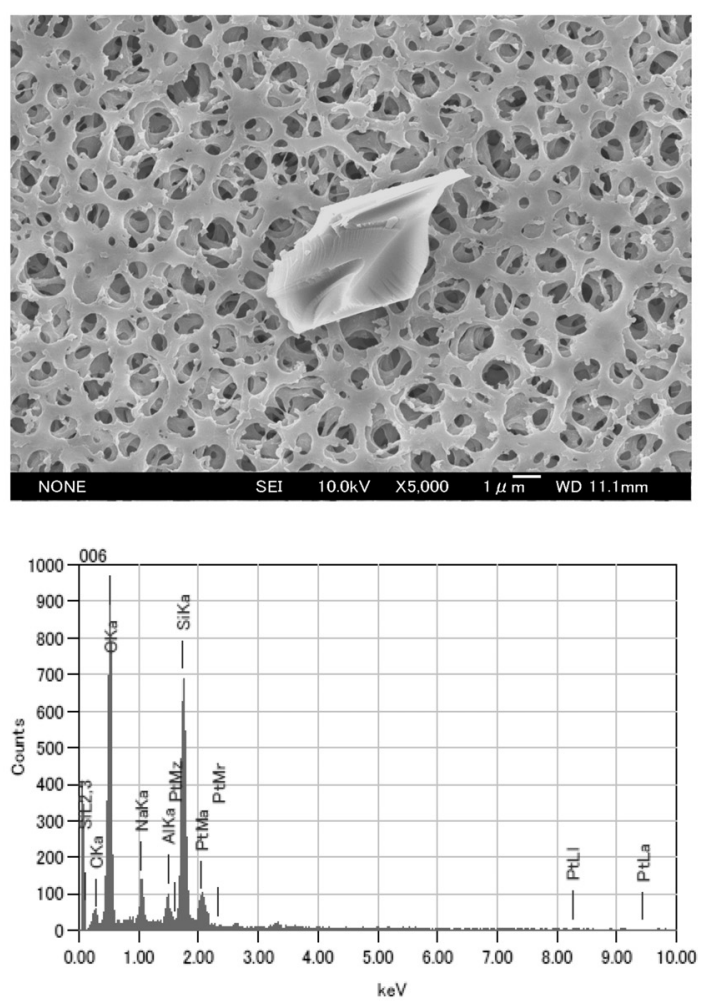

(b)
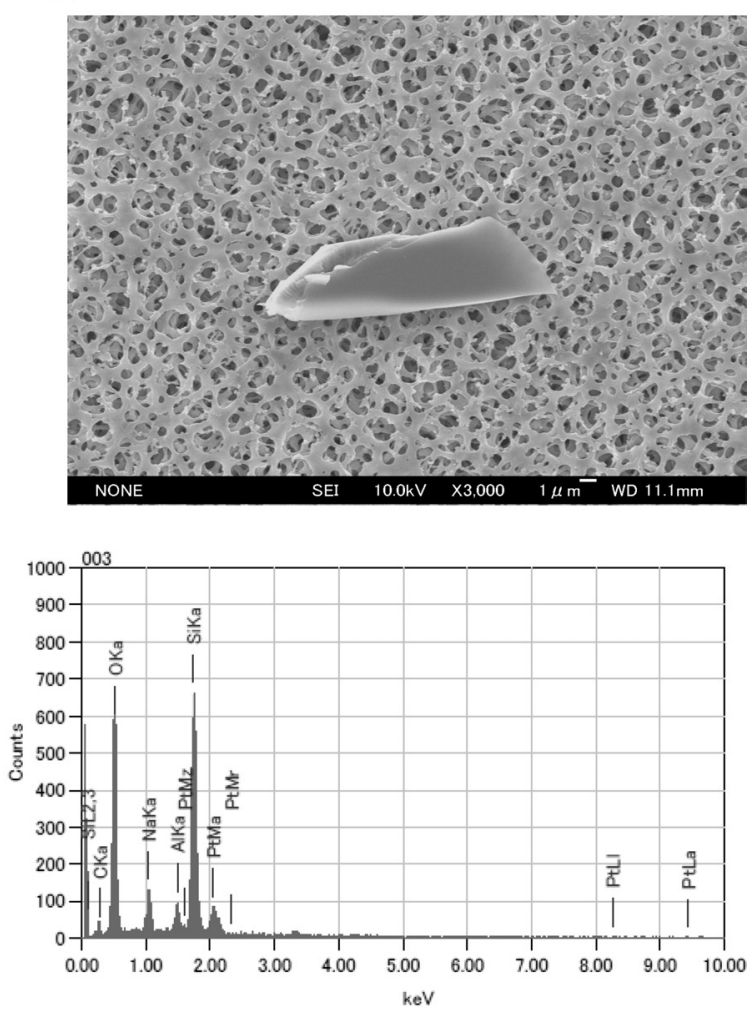

Fig. 1. Identification of Two Types of Particles by Scanning Electron Microscopy Coupled to Energy Dispersion Spectroscopy (a) and (b) suggest glass particles. 
No. $3(n=12), 117.0(68-166) / \mathrm{ml}$ for Physio ${ }^{\circledR} 35(n=11)$, $107.0(81-165) / \mathrm{ml}$ for Veen- ${ }^{\circledR} \mathrm{D}(n=10)$, and $97.2(65$ $121) / \mathrm{ml}$ for Physisalz ${ }^{\circledR}$-PL $(n=10)$.

Figure 1 shows a scanning electron micrograph of two types of particles on the filter after filtration of $5 \mathrm{ml}$ residual solution of physiological solution admixed with dobutamine hydrochloride in glass ampoules and results of the identification of these particles by energy dispersion spectroscopy. Both types of particles were glass fragments. Glass ampoule fragments were also observed in infusion solutions admixed with potassium chloride in a glass ampoule and those admixed with sodium chloride in a glass ampoule. However, no particles such as glass fragments were observed in solutions admixed with potassium chloride in a pre-filled syringe, those admixed with sodium chloride in a pre-filled syringe, or diluted dobutamine hydrochloride in pre-filled syringes.

No microbial contamination was observed in any of the 244 samples and the 66 control samples examined in this study.

\section{DISCUSSION}

The risk of the mixture of particles such as glass fragments in injection fluid at ampoule cutting has long been suggested. ${ }^{1-7)}$ In the U.S., England, and Canada, the use of glass ampoules is minimized. For example, our previous survey in Calgary University Hospital in Canada showed the use of glass ampoules only for digoxin and mesna, and predominant use of vial drugs. In Japan, though plastic ampoules for distilled water and glucose solution for injection became commercially available, ${ }^{8)}$ there have been few measures against particles in injection fluids. However, pre-filled syringes have introduced into the market, showing advantages such as saving the trouble of preparing injections, absence of the risk of injury during handling because ampoule cutting is not necessary, and a reduction in the risk of mixture of particles and microorganisms. Therefore, we compared the number of particles between infusion fluids admixed with glass ampoule drugs and those admixed with pre-filled syringe drugs, and found a significantly lower number of particles in the latter. The particles in fluids admixed with glass ampoule drugs were identified as glass fragments. These results were consistent with the presence of a large amount of glass fragments in hyperalimentation fluids admixed with glass ampoule drugs observed in our previous study. ${ }^{9}$ ) Therefore, for the prevention of mixture of particles such as glass fragments, pre-filled syringe drugs may be useful.

The sequelae of glass particle contamination require a considerable time to develop. ${ }^{5)}$ Therefore, the adverse effects of glass fragments in drugs for injection tend to be overlooked. However, glass fragments in drugs for injection have been shown to induce severe conditions such as adult distress syndrome and pulmonary artery granuloma in immature infants. ${ }^{6,7)}$ Therefore, the use of glass ampoule drugs should be also minimized in Japan. Since 12 types of drug in pre-filled syringes are commercially available at present, the use of pre-filled syringes instead of glass ampoules is desirable for these drugs. In the future, changes from glass ampoule drugs to vial drugs may be necessary as in western countries. When glass ampoule drugs are used because there is no other choice, admixtures should be prepared using membrane fil- ters at pharmacies whenever possible. ${ }^{10)}$ In the present survey, 98-299 particles (diameter, $\geq 1.3 \mu \mathrm{m}$ ) $/ \mathrm{ml}$ were detected even in residual solutions of intravenous fluids admixed with pre-filled syringe drugs. However, no particles such as glass fragments were observed in solutions admixed with drugs in pre-filled syringes. Therefore, we speculate that these particles are dust that was originally present in intravenous fluids or drugs in pre-filled syringes. These results also exclude the possibility that glass particulates come to be mixed into the solutions under experimental process to measure particulates.

The Pharmacopoeia of Japan describes the Foreign Insoluble Matter Test for Injection and Insoluble Particulate Matter Test for Injections. In the Foreign Insoluble Matter Test for Injection, drugs for injection meet the standard when no foreign matters are macroscopically observed under 8000 10000 luxes. In other words, drugs meet the standard when the diameter of contained foreign matters is less than about $40 \mu \mathrm{m}$. In the Insoluble Particulate Matter Test for Injections, the criteria are 25 or less particles with a diameter of $\geq 10 \mu \mathrm{m}$ and 3 or less particles with a diameter of $\geq 25 \mu \mathrm{m}$ per $\mathrm{ml}$. Thus, these standards refer to foreign matters and particles with a diameter of $\geq 10 \mu \mathrm{m}$. However, foreign matters and particles with a diameter of $\leq 10 \mu \mathrm{m}$ may also be harmful to the body. ${ }^{4}$ Therefore, in the future, the contents of the Foreign Insoluble Matter Test for Injection and Insoluble Particulate Matter Test for Injections in the Pharmacopoeia of Japan should be revised. In addition, these criteria in the Pharmacopoeia are used for drug products for injection, and there are no criteria for foreign matters and particles in drugs for injection after ampoule cutting. In the future, the criteria of the counts of foreign matters and particles in drugs for injection after ampoule cutting should be established for the promotion of changes in the drug form from glass ampoules to plastic ampoules or vials.

Concerning microbial contamination of peripheral intravenous admixtures, a previous study in Mexico showed microbial contamination in $16(2.13 \%)$ of 751 samples. $^{11)}$ In this study, none of the 234 samples showed microbial contamination. Therefore, the microbial contamination rate after admixture is estimated to be low, but further investigation is necessary.

\section{REFERENCES}

1) DeLuca P. P., Rapp R. P., Bivins B., Griffen W. O., Am. J. Hosp. Pharm., 32, 1001-1007 (1975).

2) Schroeder H. G., DeLuca P. P., Am. J. Hosp. Pharm., 33, 543-546 (1976).

3) Falchuk K. H., Peterson L., McNeil B. J., N. Engl. J. Med., 312, 7882 (1985)

4) Turco S. J., Davis N. M., J. Am. Med. Assoc., 217, 81-82 (1971).

5) Lye S. T., Hwang N. C., Anaesthesia, 58, 84-105 (2003).

6) Walpot H., Franke R. P., Burchard W. G., Agternkamp C., Müller F. G., Mittenmayer C., Kalff G., Anaesthesia, 38, 544-548 (1989).

7) Puntis J. W. L., Wilkins K. M., Ball P. A., Rushton D. I., Booth I. W., Arch. Dis. Child., 67, 1475-1477 (1992).

8) Oppenheim R. C., Gillies I. R., J. Pharm. Pharmacol., 38, 344-347 (1986).

9) Oie S., Kamiya A., Biol. Pharm. Bull., 28, 2268-2270 (2005).

10) American Society of Hospital Pharmacists, Am. J. Hosp. Pharm., 50, 2386-2398 (1993).

11) Macías A. E., Munoz J. M., Bruckner D. A., Rodríguez A. B., Guerrero F. J., Medina H., Gallaga L. C., Cortés G., Am. J. Infect. Control, 27, 285-290 (1999). 